

\section{ÍNDICE}

\section{ÁMBITOS PERSONALES PERSONALÁMBITOS}

Crisis de Venezuela: Análisis y perspectivas según los titulares de la prensa argentina, española y china

Venezuela's crisis: Analysis and perspectives in the headlines of Argentine, Spain and China press

Hui Feng Liu

Tratamiento informativo de la violencia de género: asesinatos de mujeres. Análisis de la agencia EFE

Informative treatment of gender violence: murders of women. Analysis of the press agency EFE Rosa Rodríguez Cárcela, Agustín López Vivas

MONOGRAFICOS MONOGRAPHS

Presentación Monográfico. El universo transmedia de los medios de comunicación universitarios: acción dentro y fuera del aula en la sociedad postdigital

Antonia Isabel Nogales-Bocio, Ángels Álvarez villa

El papel transformador de la radio universitaria en materias teóricas ajenas a la comunicación

The transformative role of college radio in theoretical subjects outside communication

Miguel Ángel Díaz Monsalvo

La radiodifusión universitaria: acción discursiva radiofónica para la divulgación de la ciencia University Radio Broadcasting: Radiophonic Discursive Action for the Science Popularization Jorge Sadi Durón, Joel Zapata Salazar

El uso corporativo de Instagram en las universidades privadas españolas. Estudio comparativo de treinta y cinco universidades

The corporate use of Instagram in spanish private universities. Comparative analysis of thirty-five private universities 
La radio universitaria como herramienta de inclusión social: OndaCampus en contextos como la cárcel y barrios desfavorecidos

The university radio as a tool for social inclusion: OndaCampus in contexts like the prison and disadvantaged neighborhoods

Leonor Real Adame, Daniel Martín-Pena, Macarena Parejo Cuéllar

Hacer radio universitaria en la era de YouTube: uso de la plataforma de vídeos a demanda en el contexto mexicano

Make college radio in the age YouTube: use of video on demand platform in the Mexican context Marina Vázquez Guerrero

\section{ARTÍCULOS ARTICLES}

O impresso e o digital nos modelos de negócios de jornais locais: uma análise do Sermos Galiza

The press and digital in business models from local media: Analysis of newspaper Sermos Galiza

Giovanni Ramos

Publicidad y cáncer en la prensa escrita (1903-1912)

Advertising and cancer in the written press (1903-1912)

Laura Almudéver-Campo, Ramón Camaño-Puig

Game rules vs. fandom. How Nintendo's Animal Crossing fan-made content negotiates the videogame meanings

Las reglas del juego vs. el fandom. Cómo el contenido hecho por fans de Nintendo Animal Crossing negocia los significados del videojuego

Jose A. Moreno

La comedia de situación y su análisis textual: evolución de los elementos constructivos del formato

The sitcom and its textual analysis: evolution of the constructive elements of the format

Darío Martín Sánchez

Análisis del discurso emocional de Donald Trump en la campaña electoral de $\mathbf{2 0 1 6}$ Analysis of Donald Trump's emotional speech on the 2016 election campaign 
Innovar, comunicar y transformar (en) la Universidad

Innovate, communicate and transform (at) the University

María Sofía Bernat

288-292

Transparencia en los medios: Un requisito imprescindible para medir la rentabilidad social en radio y televisión

Transparency in the media: An essential requirement to measuresocial profitability in radio and television

Amanda Salazar

Los estudios feministas en comunicación: representación de las mujeres en la revolución tecnológica

Feminist studies in communication: representation of women in the technological revolution 


\title{
Transparencia en los medios: Un requisito imprescindible para medir la rentabilidad social en radio y televisión
}

\author{
Transparency in the media: An essential requirement to \\ measuresocial profitability in radio and television
}

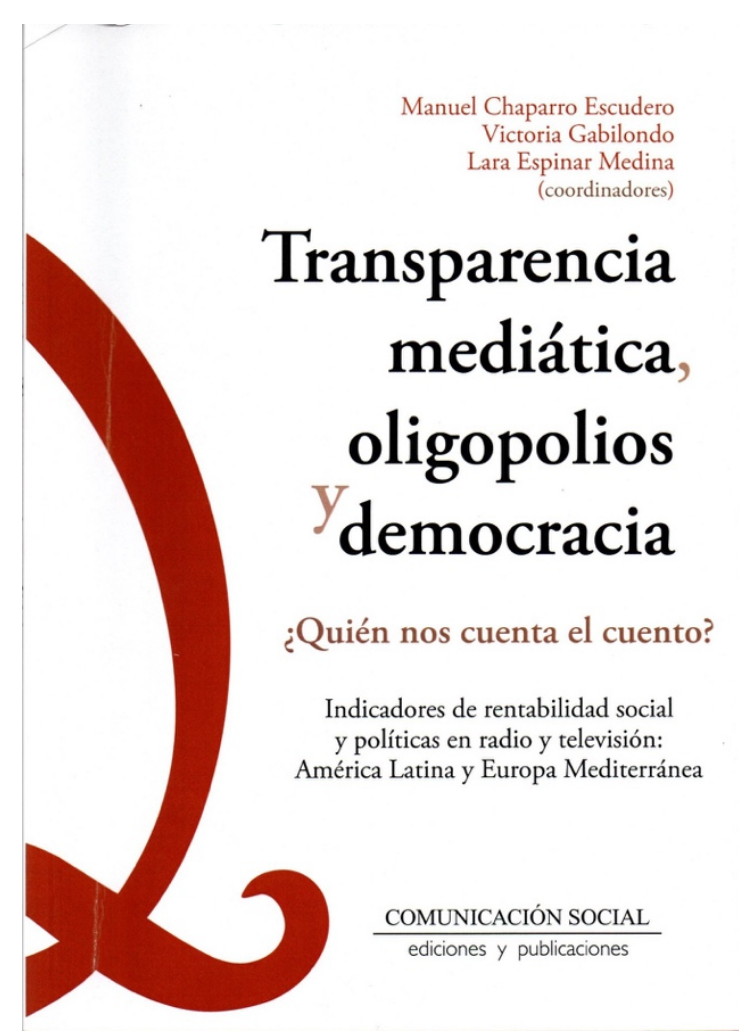

Transparencia mediática, oligopolios y democracia. ¿Quién nos cuenta el cuento? Indicadores de rentabilidad social y políticas en radio y televisión: América Latina y Europa mediterránea Chaparro Escudero, M.; Gabilondo, V.; Espinar Medina, L. (coord.)

Comunicación Social Ediciones y

Publicaciones, Salamanca, 2019 235 páginas

Reseña por Amanda Salazar

DOI: http://dx.doi.org/10.12795/Ambitos.2020.i47.15 
La transparencia democrática se ha convertido en una exigencia de la ciudadanía para velar por que la clase política desarrolle su labor pensando en el bien común y evitar que pueda actuar con otros intereses. Precisamente, los medios de información, se han convertido en los defensores a ultranza de esta transparencia, exigiéndola a instituciones públicas y a quienes ostentan cargos públicos o políticos. Pero cuando se observa el funcionamiento de las empresas periodísticas, no siempre predican con el ejemplo, son muy escasas las que están comprometidas con la transparencia empresarial como principio ético.

En un panorama tendente a la concentración, los oligopolios mediáticos buscan sus propios beneficios mientras que influyen en el poder político e intentan condicionar a la opinión pública. Esta actitud de los medios concentrados no se produce tanto por la falta de ética de los profesionales, sino por el dominio del capital que interviene en dichos medios, construyendo su agenda y mostrando una realidad a la medida de sus intereses. La concentración mediática acaba invisibilizando otras realidades y no favorece un debate profundo sobre alternativas a un modelo socio-económico desarrollista que está en crisis. En este contexto, incidir en la rentabilidad social que los medios deben revertir en la sociedad se convierte en una necesidad, sobre todo en aquellos que hacen uso del espectro radioeléctrico patrimonio del conjunto de la sociedad.

El libro coordinado por los autores Manuel Chaparro Escudero, Victoria Gabilondo García y Lara Espinar Medina, titulado Transparencia mediática, oligopolios y democracia. ¿Quién nos cuenta el cuento? Indicadores de rentabilidad social y políticas en radio y televisión: América Latina y Europa mediterránea, profundiza en una primera parte en el trabajo desarrollado en los últimos años por el Laboratorio de Comunicación y Cultura COMandalucía de la Universidad de Málaga, que ha permitido elaborar el Índice de Rentabilidad Social en Comunicación (IRSCOM) y pretende recoger los valores ligados al funcionamiento de los medios audiovisuales, midiendo factores como participación ciudadana, pluralidad, condiciones laborales y la transparencia en su gestión, entre otros aspectos.

En el año 2015, COMandalucía presentó el mapa de las radios de proximidad públicas y comunitarias, midiendo a través del IRSCOM el comportamiento de estos medios y creando, a partir de los resultados, recomendaciones en base al análisis de las fortalezas y debilidades observadas. Medidas que, según valoran los autores, han sido bien recibidas por parte de los medios en cuestión, que han empezado a introducir cambios. La propuesta del uso de indicadores ha sido, además, incluida en la Ley Audiovisual de Andalucía (2018). En este nuevo estudio, el grupo da un paso más allá 
y amplía estos indicadores a las radios comerciales generalistas y cadenas para comprobar el panorama actual.

Los autores analizan en primer lugar el contexto legislativo en España y Europa referido a los medios audiovisuales. La normativa, reforzada por la nueva Directiva Europea aprobada a finales de 2018, viene a incidir en la idea de la Directiva de Medios Audiovisuales Sin Fronteras en cuanto a la necesidad de vigilar la concentración y potenciar mecanismos de control y regulación. Pese a esto, el estudio constata la concentración de cadenas en detrimento de los medios locales en el espectro radiofónico español y el fracaso en España de la Ley General de Comunicación Audiovisual (2010).

La aplicación del IRSCOM a las radios comerciales se ha enfrentado en esta investigación a dificultades ante la opacidad y falta de transparencia de las empresas mediáticas, que no se sienten en la obligación legal de rendir cuentas sobre su funcionamiento. Por otro lado, la propia inexistencia de radios comerciales locales -en realidad son meros repetidores de señal de las cadenas generalistas y temáticas musicales privadas- ya supone de hecho la anulación de su rentabilidad social, puesto que carecen de incidencia en los territorios desde los que emiten. Pese a esto, el grupo continuó la elaboración de su investigación para aplicar parámetros de rentabilidad social a las cadenas generalistas. Las mediciones muestran la presencia dominante de tres grupos que acaparan prácticamente el espectro radiofónico comercial: Prisa, Cope y Atresmedia. Del mismo modo, el análisis de su programación muestra que estos medios han abandonado prácticamente las coberturas locales y autonómicas. Como ejemplo, se analiza en profundidad, el caso del grupo Prisa en Andalucía, pero las conclusiones son extensivas a todas las radios comerciales en cualquier comunidad autónoma de España, puesto que el comportamiento es similar en todas ellas: El modelo de programación estándar centralizada predomina en el conjunto de las emisoras y solo algunas radios locales aportan otros contenidos vinculados al territorio. La escasa rentabilidad de la radio local es parte del problema, pero está alimentado por un modelo que prima a la cadena como negocio al ignorar la medición de impacto de los contenidos de las emisoras locales y sobre todo la necesidad de estas coberturas básicas para el debate del territorio.

Una normativa laxa, tendente a la desregulación por la falta de autoridades de control independientes, ha contribuido a esta realidad. Mejorar la transparencia de estas empresas se convierte en el principal reto que deberían afrontar los medios audiovisuales analizados, más aún cuando su línea editorial exige precisamente desde una postura de denuncia un comportamiento ejemplar en estos asuntos a los gestores públicos, representantes políticos y personas físicas o jurídicas relacionadas con la 
Administración y la Ley. El resultado de la investigación muestra, en un indicador numérico, las debilidades y fortalezas de la radio comercial de cadena en España. Los grupos y las cadenas de su propiedad no salen bien paradas, y aunque se detectan fortalezas ninguna supera parámetros óptimos en el apartado de gestión y transparencia, la opacidad es extrema. De esta "formalidad" se deriva uno de los subtítulos del libro “¿quién nos cuenta el cuento?"

Las empresas propietarias de los medios deben incorporarse a las obligatoriedades de las Leyes de Transparencia, difundir sus fuentes de ingresos, los ingresos que tienen sus ejecutivos en el caso de medios que sean medios públicos. Los medios mantienen relaciones de intereses que permean el poder político y viceversa, por ello la transparencia es vital para que los ciudadanos conozcan la realidad del medio que les informa.

Por otro lado, se analiza, en otro capítulo, ampliar los indicadores que componen el IRSCOM. Hasta ahora, se analizaban cinco grandes apartados: Gestión y transparencia, capital social, articulación territorial, programación y presencia en Internet. A partir de este libro, se suma otro bloque, que surge ante la necesidad de analizar el compromiso de los medios frente a la crisis climática, como una cuestión relacionada con la economía del desarrollo y de la urgencia de trabajar las agendas para una transición ecosocial. La propuesta de Espinar, Chaparro y Peralta, recomienda la búsqueda de un modelo comunicativo que trate la realidad de manera que favorezca la transformación de un modelo ecocida. La comunicación, según defienden los autores, ya no puede permitirse ser utilizada para transferir modos, estilos de vida y hábitos de consumo que arruinan el ecosistema. La responsabilidad de los medios, que nunca debieron de alejarse del bien común, es la de trabajar en crear un nuevo modelo de sociedad.

En este punto, se propone a través del IRSCOM medir la sensibilización de los medios, si promueven el debate sobre la situación medioambiental y acompañan en la toma de conciencia ciudadana, además de visibilizar las demandas a los poderes políticos de medidas urgentes. Una apuesta que requiere una verdadera democracia mediática, dando voz a colectivos sociales que trabajan ya para este cambio.

En una segunda parte, el libro ofrece el diagnóstico de países latinoamericanos y de la Europa mediterránea, para iniciar el camino de la réplica internacional del ISRCOM junto a otros equipos de investigación asociados a los sistemas audiovisuales de Argentina, Colombia, Costa Rica, El Salvador, Nicaragua, Honduras, Guatemala, Chile, Grecia e Italia. La réplica de la experiencia busca fijar denominadores comunes que permitan 
análisis comparativos y extender este 'sello de calidad' de rentabilidad social a otros países.

La apuesta de este libro se fundamenta en la construcción de indicadores homologables internacionalmente, de referencia matemática, para poder medir comparativamente el comportamiento de los medios, algo que hasta la fecha solo han conseguido los indicadores económicos.

El reto está en establecer alianzas entre los medios de información y la ciudadanía para contribuir a la construcción social. La libertad de expresión está por encima de la libertad de empresa. Ante esta máxima, el desafío es garantizar empresas periodísticas leales a la ciudadanía que no estén sometidas a criterios mercantiles cuando nos encontramos ante una crisis del sistema de consecuencias extremas. 